\title{
Assessment of the contribution of morbidity and mortality conferences to quality and safety improvement: a survey of participants' perceptions
}

André Lecoanet ${ }^{1}$, Gwenaëlle Vidal-Trecan 2,3,4, Frédéric Prate ${ }^{5}$, Jean-François Quaranta ${ }^{5}$, Elodie Sellier ${ }^{1,6}$, Alizé Guyomard', Arnaud Seigneurin ${ }^{1,6}$ and Patrice François ${ }^{1,6^{*}}$

\begin{abstract}
Background: Evidence for the effectiveness of the morbidity and mortality conferences in improving patient safety is lacking. The aim of this survey was to assess the opinion of participants concerning the benefits and the functioning of morbidity and mortality conferences, according to their organizational characteristics.

Methods: We conducted a survey of professionals involved in a morbidity and mortality conference using a self-administered questionnaire in three French teaching hospitals in 2012. The questionnaire focused on the functioning of morbidity and mortality conferences, the perceived benefits, the motivations of participants, and how morbidity and mortality conferences could be improved. The perception of participants was analysed according to the characteristics of morbidity and mortality conferences.

Results: A total of 698 participants in 54 morbidity and mortality conferences completed the questionnaire. Most of them (91\%) were satisfied with how the morbidity and mortality conference they attended was conducted. The improvements in healthcare quality and patient safety were the main benefits perceived by participants. Effectiveness in improving safety was mainly perceived when cases were thoroughly analysed (adjusted odds ratio $[\mathrm{aOR}]=2.31[1.14-4.66])$. The existence of a written charter $(p=0.05)$, the use of a standardized case presentation $(p=0.049)$, and prior dissemination of the meeting agenda $(p=0.02)$ were also associated with the perception of morbidity and mortality conference effectiveness. The development and achievement of improvement initiatives were associated with morbidity and mortality conferences perceived as being more effective $(p<0.01)$. Participants suggested improving the attendance of medical and paramedical professionals to enhance the effectiveness of morbidity and mortality conferences.
\end{abstract}

Conclusions: Morbidity and mortality conferences were positively perceived. These results suggest that a structured framework and thoroughly analyzing cases improve their effectiveness.

Keywords: Morbidity and mortality conferences, Patient safety, Quality improvement, Hospital

\section{Background}

Morbidity and mortality conferences (MMCs) were established in the United States at the beginning of the 20th century and have become a relevant part of physician education [1]. They were initially set up by surgeons and

\footnotetext{
* Correspondence: pfrancois@chu-grenoble.fr

${ }^{1}$ Public Health Department, University Hospital, Grenoble F-38043, France ${ }^{6}$ Research Unit TIMC-IMAG (UMR 5525 CNRS/UJF-Grenoble 1), Grenoble F-38041, France

Full list of author information is available at the end of the article
}

anaesthesiologists and became a way to fulfill the mandatory requirement for assessment of physicians' practices. With the emergence of the management of patient safety in the healthcare system in the 1990s, several authors suggested that MMCs could be conducted to improve the quality and safety of healthcare [2]. In this context, MMCs were adopted in France. In 2010, the French health authorities decided to make MMCs mandatory in high-risk specialties such as surgery, anaesthesia, intensive 
care and oncology. Fulfilling this commitment was a key factor in the accreditation of hospitals.

Therefore, MMCs are now widely implemented in hospitals, but evidence of their effectiveness in improving patient safety is lacking. Numerous authors have found that the characteristics of MMCs varied greatly [3-5] with variations in their goals, their structures, and their processes.

The best evidence of the effectiveness of MMCs would be to demonstrate an effect on a patient-related outcome. However, this approach presents methodological issues and very few studies have concluded in a significant effect of MMCs on a clinical outcome [6-8]. Considering the wide variety of situations, choosing and using a patient-related outcome is difficult. Where this is possible, the low incidence of specific events would lead to a lack of statistical power [9]. In this context, several authors estimated the effectiveness of MMCs indirectly through the perception of the participants in these meetings $[4,10,11]$. They assessed their functioning and their impact in terms of knowledge or improvement of practices. Some authors emphasized the wide variety of leadership structures and the perceived effectiveness [4]. They suggested that a formalized framework should lead to a better perception of effectiveness and more satisfying MMCs $[4,10,11]$.

The present survey focused on the functioning of MMCs, the perception of their benefits by the participants, especially concerning healthcare quality and safety improvement, the personal motivations of participants, and suggestions for improvement. The main objective was to assess the participants' opinion concerning the effectiveness of MMCs as a tool to improve patient safety. Within a secondary objective, we identified the characteristics of MMC functioning associated with the perception that the MMC produced benefits.

\section{Methods}

\section{Study design}

This observational study was conducted in three teaching hospitals in France: Grenoble, Nice, and Cochin (within the Paris Hospital network) in 2012 and was based on self-administered questionnaires.

\section{Population}

The MMCs, established for more than 1 year, were identified between September and December 2010 in each hospital. Every professional involved in one of these MMCs who agreed to participate was included. The protocol of the study was first described to all MMC leaders. It was then presented and discussed within each MMC and the team agreed to the collection of information from all participants to assess their opinion on the MMC. According to the French law, no ethics review was necessary for this study considering that there was no intervention or collection of personal data [12].

\section{Data collection}

The questionnaire was based on the main items used by Aboumatar et al. to interview the MMC leaders at the John Hopkins Hospital [4]. A total of 26 multiple-choice questions explored the perception of the benefits of the $\mathrm{MMC}$, the functioning of the MMC, personal motivations, and possible improvements. Response choices followed a four-modality Likert scale from "entirely disagree" to "entirely agree". A free comment field ended the questionnaires.

The questionnaire was provided to any professional who attended at least one MMC meeting. They were delivered electronically by email or by hand at the end of a meeting. In case of electronic delivery, a reminder was sent if no reply was recorded within 15 days.

The characteristics of MMCs were collected in a previous study [13] by analyzing all the documents produced by MMCs (i.e., charter or organizational procedure, meeting reports, annual activity report, and documents related to implemented improvement initiatives) and through the observation of one meeting per MMC. The main characteristics collected were related to formalization (the presence of a written charter, an annual activity report, a list of participants and a meeting schedule, the exhaustiveness of the meeting minutes, the prior dissemination of the meeting agenda, the standardized case presentation using slides), the analysis of cases (the thoroughness of the analysis of the failure, the number of cases presented, the length of the meetings, the practice of thematic meetings), the attendance rate of occupational groups and generalities (time the MMC had existed, department specialty). The thoroughness of the analysis of the failure was coded as "0 - failures were not examined, 1 - failures were examined, 2 - failures were thoroughly analysed searching for causes". Other categorical characteristics were considered as present or absent.

Furthermore, each improvement initiative decided during the study period was followed for 1 year. The implementation of initiatives and the impact were assessed on the basis of MMC leaders' interviews and document analysis. Assessment was carried out using a score from 1 to 6 , according to the designation of a person in charge (0-1), the definition of a timeline (0-1), the completion of each initiative (0-1-2) and its evaluation (0-1-2). These scores were aggregated to obtain an effectiveness index for each MMC.

\section{Data analysis}

Descriptive statistics were performed using numbers and percentages. Ordinal and continuous variables, especially 
the effectiveness index and the number of improvement initiatives, were dichotomized according to their median.

We compared the proportion of agreement according to the main characteristics of MMC operation, the number of improvement initiatives and the effectiveness index. With an expected high rate of "at least agree" responses, we defined the agreement level as "totally agree" in order to avoid a ceiling effect.

Univariate analysis was performed using Fisher exact tests. A multivariable analysis was carried out to identify the independent characteristics associated with the perception of patient safety improvements. A mixed effect logistic regression was used to take into account the multilevel structure of the data with individuals attending MMCs. The characteristics associated with outcomes in univariate analysis with a $p$-value less than 0.2 were entered in the model. The number of actions undertaken and the effectiveness index were excluded because they reflected the effectiveness of the MMC rather than an operational characteristic. Two-sided $p$-values of less than 0.05 were considered statistically significant. Analyses were performed using STATA 11.0 software.

The free comments were analysed by two independent investigators. The content of each comment was first classified according to three categories: positive comments, negative comments, and suggestions. Secondly, each part of the comments was organized by theme. Differences in categories and themes were discussed until a consensus was reached.

\section{Results}

We identified 1134 professionals involved in $54 \mathrm{MMC}$ groups, including $24 \mathrm{MMCs}$ in medical units, 20 in surgical units, and 10 in anaesthesiology/intensive care units. Median time MMCs had existed was 4 years (IQR, $3-5)$. A total of 698 (61.6\%) participants completed the questionnaire: 395 (58 \%) senior practitioners, 158 (23.2\%) residents, 60 (8.8\%) head nurses, 28 (4.1\%) nurses, 24 (3.5 \%) midwives, and 16 other paramedical or administrative professionals. The type of occupation was missing for 17 respondents. Two hundred eighty $(40.1 \%)$ worked in a medical unit, $219(31.9 \%)$ in a surgical unit, and 199 (28.5\%) in an anaesthesiology/intensive care unit. Response rates were similar for the different specialties $(62.5 \%$ in medicine, $62.5 \%$ in surgery, $59.1 \%$ in anaesthesiology/intensive care, $p=0.55$ ).

Most of the respondents agreed totally or partially that the meeting was managed satisfactorily $(89.9 \%, n=617)$ and was non-blaming $(86.1 \%, n=589)$. More than $80 \%$ of the respondents agreed totally or partially for each benefit proposed, except improvement of the relationship between medical and paramedical teams $(60.6 \%$, $n=421)$.
Considering only respondents who totally agreed, the perceived benefits were mainly quality of care improvement and patient safety improvement (Table 1 ). Personal motivations to participate in MMCs were also most often related to improvement of individual and team practices. Only a minority of participants totally agreed with the suggested areas for improvement. The highest proportion of agreement concerned the increase of paramedical professionals' participation. There were significant differences in opinion of the various occupational groups, especially for the perceived benefits. Senior physicians and paramedical professionals had a better opinion of the benefits than did residents. Personal motivations also differed with senior physicians more often motivated by improvements in practices than residents. Senior physicians more frequently agreed that MMCs were associated with an educational aspect for initial and continuing education. They more often suggested improving the case analysis method. Paramedical staff more often acknowledged the benefits in terms of practices and improvements of organizational and relational aspects. Paramedical personnel also more often agreed with increasing the participation of the paramedical staff.

There were also differences in the perceived benefits of MMCs, depending on the specialty of the department. MMCs in medical specialties had a greater perceived impact on the relationship between medical and paramedical teams (29.5\% versus $20.9 \%, p=0.03)$. Surgical MMCs had a greater perceived impact on initial education $(45.8 \%$ versus $33.3 \%, p<0.01)$. Participants in MMCs in anaesthesia/intensive care units were less likely to consider their MMC as non-blaming (27.7\% versus $38.2 \%, p=0.03$ ).

Considering how long MMCs had existed, there were no differences in terms of perceived benefits and motivations. However, participants in the oldest MMCs were less likely to consider their MMC as non-blaming (50.2\% versus $59.1 \%, p=0.03$ ).

The characteristics of MMCs significantly associated with a better opinion were the prior dissemination of the meeting agenda, the existence of a written charter, the use of a standardized case presentation, the examination of failures during the discussion, and fewer cases presented (Table 2). In addition, a better opinion was significantly associated with a higher effectiveness index based on planning, implementation, and assessment of improvement initiatives.

A greater perception of benefits in terms of continuous training was associated with the use of standardized case presentations ( $48.4 \%$ versus $34.3 \%$, $p<0.01)$, a higher effectiveness index $(48.2 \%$ versus 
Table 1 Proportion of agreement for each question according to the professional status of MMC participants

\begin{tabular}{|c|c|c|c|c|c|}
\hline & $\begin{array}{l}\text { Total } \\
N=698^{\mathrm{a}}(\%)\end{array}$ & $\begin{array}{l}\text { Senior physicians } \\
N=395 \text { (\%) }\end{array}$ & $\begin{array}{l}\text { Residents } \\
N=158 \text { (\%) }\end{array}$ & $\begin{array}{l}\text { Others }^{b} \\
N=128(\%)\end{array}$ & $p$ \\
\hline \multicolumn{6}{|l|}{ Perceived benefits } \\
\hline Initial education & 37.0 & 42.3 & 30.4 & 28.6 & 0.004 \\
\hline Continuing education & 42.4 & 50.0 & 31.0 & 35.1 & $<0.001$ \\
\hline Improvement of quality of care & 61.9 & 66.8 & 51.3 & 67.2 & 0.002 \\
\hline Improvement of patient safety & 63.2 & 67.8 & 52.5 & 68.7 & 0.002 \\
\hline Standardization of medical practices & 45.2 & 47.8 & 27.8 & 60.8 & $<0.001$ \\
\hline Application of clinical guidelines & 37.2 & 37.9 & 26.3 & 52.1 & $<0.001$ \\
\hline Improvement of functioning in the unit & 47.7 & 50.8 & 35.0 & 55.1 & 0.001 \\
\hline Improvement of teamwork & 33.0 & 36.5 & 17.7 & 43.8 & $<0.001$ \\
\hline Improvement of relations between medical and paramedical teams & 22.8 & 21.9 & 12.7 & 42.5 & $<0.001$ \\
\hline Improvement of safety culture & 35.9 & 39.4 & 22.1 & 44.7 & $<0.001$ \\
\hline Discussion of collective errors & 54.8 & 55.2 & 44.3 & 67.2 & 0.001 \\
\hline \multicolumn{6}{|l|}{ Functioning assessment } \\
\hline Friendly environment & 40.1 & 44.8 & 29.7 & 38.2 & 0.004 \\
\hline Avoidance of blame & 35.9 & 38.4 & 29.9 & 33.3 & 0.15 \\
\hline Satisfactory conduct & 34.1 & 40.4 & 24.4 & 37.6 & 0.002 \\
\hline \multicolumn{6}{|l|}{ Personal motivations } \\
\hline Fulfillment of mandatory training requirement & 12.2 & 12.7 & 12.3 & 9.9 & 0.78 \\
\hline Social interactions & 15.7 & 17.1 & 11.5 & 18.8 & 0.17 \\
\hline Improve professional practices & 71.6 & 75.9 & 58.0 & 69.0 & $<0.001$ \\
\hline Improve team practices & 75.6 & 82.2 & 56.3 & 74.6 & $<0.001$ \\
\hline improve of functioning in the unit & 66.1 & 72.3 & 50.6 & 68.5 & $<0.001$ \\
\hline \multicolumn{6}{|l|}{ Areas for improvement } \\
\hline Increasing the number of meetings & 6.7 & 6.2 & 7.6 & 4.2 & 0.50 \\
\hline Increasing the participation of senior physicians & 22.6 & 24.0 & 15.4 & 22.0 & 0.10 \\
\hline Increasing the participation of the unit head & 16.2 & 18.1 & 10.8 & 17.8 & 0.10 \\
\hline Increasing the participation of invited specialists & 26.4 & 29.5 & 22.9 & 21.0 & 0.10 \\
\hline Increasing the participation of paramedical professionals & 31.9 & 31.0 & 21.5 & 42.6 & 0.001 \\
\hline Improving the case selection method & 16.4 & 17.1 & 12.8 & 12.9 & 0.36 \\
\hline Improving the case analysis method & 14.6 & 17.2 & 7.6 & 12.1 & 0.01 \\
\hline
\end{tabular}

${ }^{a}$ Includes 17 participants whose occupation was missing

${ }^{\mathrm{b}}$ Includes midwives, nurses, head nurses, and other paramedical professionals

Agreement is defined as "totally agree" response

$36.8 \% \%, p<0.01)$, and more decisions on initiatives ( $47.2 \%$ versus $35.2 \%, p<0.01$ ).

Participants in MMCs examining failures, without analysis, were significantly less likely to consider their MMC as non-blaming compared to those participating in MMCs that did not examine failures $(34.0 \%$ versus $49.1 \%, p=0.03)$. However, this difference tended to disappear when the failures were accurately analysed ( $36.7 \%$ versus $34.54 \%, \mathrm{p}=0.60$ ).

Participants from MMCs with a greater effectiveness index more frequently perceived benefits in terms of continuing education ( $48.2 \%$ versus $36.8 \%, p<0.01$ ), standardization of practices (51.3\% versus $39.4 \%$, $p<0.01)$, and application of guidelines $(41.6 \%$ versus $33.3 \%, p<0.01)$.

In multivariable analysis, a thorough analysis of the causes of failures was independently associated with a greater perception of improvement of patient safety (Table 3).

A total of 110 respondents $(15.8 \%)$ wrote a comment after having answered the structured questionnaire. Fourteen highlighted the value of MMCs and three others underscored the high rate of physician attendance. Most of them (31) emphasized a low participation 
Table 2 Proportion of agreement with the perception of patient safety improvement, according to MMC operational characteristics

\begin{tabular}{|c|c|c|c|}
\hline & \multicolumn{3}{|c|}{ MMCs contribute to improving patient safety } \\
\hline & If characteristic is absent $N^{a}(\%)$ & If characteristic is present $N^{a}(\%)$ & $p$ \\
\hline \multicolumn{4}{|l|}{ Formalization } \\
\hline Written charter & $38(53.6)$ & $412(65.8)$ & 0.049 \\
\hline Yearly activity report & $76(59.8)$ & $374(65.6)$ & 0.22 \\
\hline List of participants & $76(63.9)$ & $374(64.7)$ & 0.92 \\
\hline Exhaustiveness of meeting minutes & $57(62.64)$ & $393(64.9)$ & 0.73 \\
\hline Prior dissemination of meeting agenda & $156(59.1)$ & $291(68.0)$ & 0.02 \\
\hline Established meeting schedule & $248(65.4)$ & $202(63.5)$ & 0.63 \\
\hline Standardized presentation using slides & $153(57.9)$ & $297(68.6)$ & 0.005 \\
\hline \multicolumn{4}{|l|}{ Content, analysis } \\
\hline \multicolumn{4}{|l|}{ Failures are } \\
\hline examined & $24(42.1)$ & $426(66.6)$ & $<0.001$ \\
\hline and thoroughly analysed & $292(61.3)$ & $158(71.5)$ & 0.01 \\
\hline Few cases presented $(<19)$ & $206(59.2)$ & $244(69.9)$ & 0.03 \\
\hline Longer meetings (>80 min) & $234(63.9)$ & $216(65.3)$ & 0.75 \\
\hline Thematic meetings organized & $359(63.0)$ & $91(71.6)$ & 0.07 \\
\hline \multicolumn{4}{|l|}{ Attendance rates (if greater than the median) } \\
\hline Senior physicians & $226(64.2)$ & $224(64.9)$ & 0.87 \\
\hline Head nurses & $255(63.6)$ & $195(65.9)$ & 0.57 \\
\hline Nurses & $195(62.1)$ & $255(66.6)$ & 0.23 \\
\hline Older group ( $\geq 4$ years of experience) & $198(65.13)$ & $252(64.12)$ & 0.81 \\
\hline \multicolumn{4}{|l|}{ Improvement initiatives } \\
\hline Effectiveness index $(\geq 10)^{b}$ & $185(59.5)$ & $265(68.6)$ & 0.01 \\
\hline Number of initiatives (>2) & $144(60.8)$ & $306(66.5)$ & 0.13 \\
\hline
\end{tabular}

${ }^{a}$ Count of "totally agree" responses

${ }^{\mathrm{b}}$ The effectiveness index was a composite score based on planning, implementation, and assessment of improvement initiatives

rate for several professional categories, especially anaesthetists in surgical wards and paramedical professionals. Another topic related to improvement of case selection with 14 comments. In particular, ten participants suggested reviewing more relevant cases. Concerning the organization of MMCs, 12 comments highlighted the lack of time available for healthcare professionals. Four

Table 3 Multivariable analysis of safety improvement perception according to MMC operational characteristics

\begin{tabular}{lll}
\hline & Adjusted OR [95 \% CI] & $p$ \\
\hline Written charter & $1.30[0.72-2.36]$ & 0.38 \\
Prior dissemination of meeting agenda & $1.10[0.74-1.62]$ & 0.63 \\
More cases presented & $0.76[0.53-1.10]$ & 0.15 \\
Thematic MMC & $1.25[0.76-2.08]$ & 0.38 \\
Standardized presentation & $1.14[0.77-1.68]$ & 0.51 \\
Failures are & & \\
$\quad$ examined & $1.85[0.96-3.55]$ & 0.06 \\
$\quad$ and thoroughly analysed & $2.46[1.20-5.03]$ & 0.01 \\
\hline
\end{tabular}

professionals described an open and friendly environment, whereas eight described a blameful environment with conflicts and subjects that could not be debated.

Finally, a lack of a structured analysis of cases was reported. According to the respondents, the follow-up of initiatives should be improved as well as information provided outside the committee. A lack of involvement from the hospital administration was also pointed out.

\section{Discussion}

Participants mostly agreed that MMCs were beneficial in terms of healthcare quality and safety improvement. MMCs were perceived as beneficial for teamwork and the functioning of the unit. The improvement of practices and organization seemed to be the main objective perceived by the participants. Moreover, an educational role, for initial and continuing education, was perceived by most participants, particularly by the senior physicians. Most of participants were satisfied with MMCs and experienced a friendly and nonblaming environment. 
The search for failures and the discussion of errors are well-documented opportunities for improvement of safety and education [2, 14]. The present results suggest that the analysis of these failures was perceived as determinant in improving patient safety. However, without a thorough analysis, this discussion seems to result in a blaming environment that is incompatible with constructive discussion [6, 15-18]. Indeed, personal failures are often spontaneously discussed first. To overcome personal accusation and to develop a system-wide approach, a thorough analysis should be carried out using a systematic analysis method such as the protocol of the Association of Litigation and Risk Management (ALARM) [19]. However, due to the low number of MMCs that used a systematic method of analysis, it was not possible to study its impact. These methods are known and described, but it must be recognized that they are not commonly used [20]. The time constraints described by several professionals could be a reason for the underuse of a time-consuming method of analysis.

Moreover, the number and the type of cases discussed varied and affected the accuracy of analyses. Some authors suggest reviewing all deaths and complications to avoid missing opportunities to identify failures [21-23]. Others suggest performing a more thorough analysis based on a prior selection of relevant cases [4, 11, 14]. As the opportunity to spend more time on cases was positively perceived in this study, we would favor this method. According to numerous authors, the choice of cases should be based on errors or system safety issues $[2,16,24]$.

The characteristics related to a formalized framework were associated with the perception of MMCs being more effective. This result is consistent with the literature and particularly with the impact of standardized presentations on participant satisfaction and on the production of improvement initiatives $[10,11]$. This type of formalized framework could be valuable in providing more time for a system-oriented discussion [16].

The participants suggested including other professionals such as paramedical staff in the MMCs. Considering that incidents could involve all the healthcare professionals in a medical unit, this would allow a more accurate and system-oriented analysis $[4,6,17$, 25, 26] and increase the acceptance of improvement initiatives [16, 26]. Furthermore, paramedical professionals take an interest in safety management [27]. Such a team-based improvement meeting is also a way to establish a culture of safety within the department $[16,26]$.

We note that residents perceived fewer benefits than the other professionals. Our results do not suggest that they perceived MMCs as more blaming. Given that they change units every 6 months, they cannot perceive longterm improvement. Moreover, they are undoubtedly more focused on their personal training and therefore less motivated by team improvement.

A higher effectiveness index was associated with a greater perception of the effectiveness of MMCs in terms of patient safety. This suggests that an index based on the implementation of improvement initiatives could be a valuable way to assess the effectiveness of MMCs, although this hypothesis deserves further investigation based on clinical outcomes. The type of improvement initiatives and their follow-up are also relevant [28]. Moreover, the results of this study are consistent with those obtained previously concerning the characteristics associated with more effective MMCs.(P François, et al., submitted manuscript).

This study had several limitations. First, we assessed MMCs using the participants' subjective perceptions. However, these perceptions are associated with the effectiveness index, a more objective measure of effectiveness. Second, we cannot exclude a selection bias and respondents could be particularly motivated or, on the other hand, more critical. Finally, this study was conducted in only three French hospitals. The development of MMCs is rather recent in France and they sometimes take place within specific settings. Consequently, the conclusions should be considered with regard to these specificities.

\section{Conclusions}

In conclusion, this study showed the participants' interest in MMCs. A thorough analysis of failures stands out as the main beneficial characteristic. The suggestions for improving MMCs concern a more structured framework and the attendance by a greater variety of professional categories in order to guide MMCs toward a systemwide approach. Furthermore, the improvement initiatives were associated with MMCs perceived as being more effective.

\section{Competing interests}

The authors declare that they have no competing interest.

\section{Authors' contributions}

$\mathrm{AL}$ analysed and interpreted data and drafted the manuscript. GVT participated in the design of the work and revised the manuscript for important intellectual content. FP: participated in the design of the work and revised the manuscript for important intellectual content. JFQ participated in the design of the work and revised the manuscript for important intellectual content. ES participated in the data analysis and interpretation and revised the manuscript for important intellectual content. AG participated in data acquisition, management and analysis and revised the manuscript for important intellectual content. AS participated in the data analysis and interpretation and revised manuscript for important intellectual content. PF participated in the design of the work, in data analysis and revised the manuscript for important intellectual content. All authors approved the final version to be published agreed to be accountable for all aspects of the work. 


\section{Acknowledgments}

We acknowledge Linda Northrup for her assistance in editing the manuscript in English.

We acknowledge José Labarère for his statistical advice.

\section{Funding}

This study was supported by the French Ministry of Health: Projet de Recherche en Qualité Hospitalière [25Preghos10]. The French Ministry of Health did not participate in design or conduct of the study; collection, management, analysis, and interpretation of the data; or in preparation, review, or approval of the manuscript.

\section{Author details}

${ }^{1}$ Public Health Department, University Hospital, Grenoble F-38043, France. ${ }^{2}$ Public health unit: Risk Management and Quality of Care, Paris Centre University Hospital Group, AP-HP, Paris F-75014, France. ${ }^{3}$ Research Unit: METHODS team, INSERM U1153 (Centre de Recherche Epidémiologie Biostatistique, Sorbonne Paris Cité), Paris F-75005, France. ${ }^{4}$ Medical School, Paris Descartes University, Paris F-75006, France. ${ }^{5}$ Public Health Department, University Hospital, Nice F-06003, France. ${ }^{6}$ Research Unit TIMC-IMAG (UMR 5525 CNRS/UJF-Grenoble 1), Grenoble F-38041, France.

\section{Received: 15 October 2014 Accepted: 5 February 2016}

\section{Published online: 11 May 2016}

\section{References}

1. Kravet SJ, Howell E, Wright SM. Morbidity and mortality conference, grand rounds, and the ACGME's core competencies. J Gen Intern Med. 2006;21:1192-4

2. Pierluissi E, Fischer MA, Campbell AR, Landefeld CS. Discussion of medical errors in morbidity and mortality conferences. JAMA. 2003:290:2838-42.

3. Orlander JD, Fincke BG. Morbidity and mortality conference: a survey of academic internal medicine departments. J Gen Intern Med. 2003;18:656-8.

4. Aboumatar HJ, Blackledge CG, Dickson C, Heitmiller E, Freischlag J, Pronovost PJ. A descriptive study of morbidity and mortality conferences and their conformity to medical incident analysis models: results of the morbidity and mortality conference improvement study, phase 1. Am J Med Qual. 2007:22:232-8.

5. Bal G, Sellier E, Tchouda SD, François P. Improving quality of care and patient safety through morbidity and mortality conferences. J Healthc Qual. 2012;36:29-36.

6. Kirschenbaum L, Kurtz S, Astiz M. Improved clinical outcomes combining house staff self-assessment with an audit-based quality improvement program. J Gen Intern Med. 2010;25:1078-82.

7. Papiernik E, Bucourt M, Zeitlin J. Measuring the success of the audit process: results from the Seine-Saint-Denis perinatal audit. Prenat Neonatal Med. 2000:5:347-54

8. Dupont C, Deneux-Tharaux C, Touzet S, Colin C, Bouvier-Colle M-H, Lansac J, Thevenet S, Boberie-Moyrand C, Piccin G, Fernandez M-P, Rudigoz R-C. Clinical audit: a useful tool for reducing severe postpartum haemorrhages? Int J Qual Health Care. 2011:23:583-9.

9. Denis B, Ben Abdelghani M, Peter A, Weiss A-M, Bottlaender J, Goineau J. [Two years of mortality and morbidity conferences in a hospital gastrointestinal endoscopy unit]. Gastroenterol Clin Biol. 2003;27:1100-4

10. Kim MJ, Fleming FJ, Peters JH, Salloum RM, Monson JR, Eghbali ME. Improvement in educational effectiveness of morbidity and mortality conferences with structured presentation and analysis of complications. J Surg Educ. 2010;67:400-5.

11. Risucci DA, Sullivan T, DiRusso S, Savino JA. Assessing educational validity of the Morbidity and Mortality conference: a pilot study. Curr Surg. 2003:60:204-9.

12. Republic F. Loi $n^{\circ} 2012-300$ du 5 mars 2012 relative aux recherches impliquant les personnes humaines. Journal officiel de la république française. 2012;56:10-8

13. François P, Prate F, Vidal-Trecan G, Quaranta JF, Labarere J, Sellier E. Characteristics of morbidity and mortality conferences associated with the implementation of patient safety improvement initiatives, an observationa study. BMC health services research, 2016;16(1):1
14. Gore DC. National survey of surgical morbidity and mortality conferences. Am J Surg. 2006;191:708-14.

15. Epstein NE. Morbidity and mortality conferences: Their educational role and why we should be there. Surg Neurol Int. 2012;3 Suppl 5:S377-88.

16. Deis JN, Smith KM, Warren MD, Throop PG, Hickson GB, Joers BJ, Deshpande JK. Transforming the Morbidity and Mortality Conference into an Instrument for Systemwide Improvement. In: Henriksen K, Battles J, Keyes M, Grady M, editors. Adv Patient Saf New Dir Altern Approaches (Vol 2 Cult Redesign). Rockville: Agency for Healthcare Research and Quality (US); 2008.

17. Sellier E, David-Tchouda S, Bal G, François P. Morbidity and mortality conferences: their place in quality assessments. Int J Health Care Qual Assur. 2012;25:189-96

18. Leape LL. Reporting of adverse events. N Engl J Med. 2002;347:1633-8.

19. Vincent C, Taylor-Adams S, Chapman EJ, Hewett D, Prior S, Strange P, Tizzard A. How to investigate and analyse clinical incidents: clinical risk unit and association of litigation and risk management protocol. BMJ. 2000;320:777-81.

20. Bal G, David S, Sellier E, François P. [Value of morbidity and mortality review conferences for physician education and improvement of care quality and safety: a literature review]. Presse Med. 2010;39:161-8.

21. Murayama KM, Derossis AM, DaRosa DA, Sherman HB, Fryer JP. A critical evaluation of the morbidity and mortality conference. Am J Surg. 2002;183:246-50.

22. Hutter MM, Rowell KS, Devaney LA, Sokal SM, Warshaw AL, Abbott WM, Hodin RA. Identification of surgical complications and deaths: an assessment of the traditional surgical morbidity and mortality conference compared with the American College of Surgeons-National Surgical Quality Improvement Program. J Am Coll Surg. 2006;203:618-24.

23. Tighe CM, Woloshynowych M, Brown R, Wears B, Vincent C. Incident reporting in one UK accident and emergency department. Accid Emerg Nurs. 2006:14:27-37.

24. Bechtold ML, Scott S, Dellsperger KC, Hall LW, Nelson K, Cox KR. Educational quality improvement report: outcomes from a revised morbidity and mortality format that emphasised patient safety. Postgrad Med J. 2008;84:211-6

25. Schwarz D, Schwarz R, Gauchan B, Andrews J, Sharma R, Karelas G, Rajbhandari R, Acharya B, Mate K, Bista A, Bista MG, Sox C, Smith-Rohrberg Maru D. Implementing a systems-oriented morbidity and mortality conference in remote rural Nepal for quality improvement. BMJ Qual Saf. 2011:20:1082-8

26. Kauffmann RM, Landman MP, Shelton J, Dmochowski RR, Bledsoe SH, Hickson GB, Beauchamp RD, Dattilo JB. The use of a multidisciplinary morbidity and mortality conference to incorporate ACGME general competencies. J Surg Educ 2011;68:303-8

27. Lecoanet A, Sellier E, Carpentier F, Maignan M, Seigneurin A, François P. Experience feedback committee in emergency medicine: a tool for security management. Emerg Med J. 2014;31:894-8

28. Wu AW, Lipshutz AKM, Pronovost PJ. Effectiveness and efficiency of root cause analysis in medicine. JAMA. 2008:299:685-7.

\section{Submit your next manuscript to BioMed Central and we will help you at every step:}

- We accept pre-submission inquiries

- Our selector tool helps you to find the most relevant journal

- We provide round the clock customer support

- Convenient online submission

- Thorough peer review

- Inclusion in PubMed and all major indexing services

- Maximum visibility for your research

Submit your manuscript at www.biomedcentral.com/submit
Biomed Central 